\title{
Panoramic Views for Virtual Endoscopy
}

\author{
Bernhard Geiger, Christophe Chefd'hotel, and Sandra Sudarsky \\ Siemens Corporate Research Inc., Princeton, NJ, 08540, USA \\ \{geiger.bernhard, christophe.chefdhotel, sandra.sudarsky\}@siemens.com
}

\begin{abstract}
This paper describes a panoramic projection designed to increase the surface visibility during virtual endoscopies. The proposed projection renders five faces of a cubic viewing space into the plane in a continuous fashion. Using this real-time and interactive visualization technique as a screening method for colon cancer could lead to significantly shorter evaluation time. It avoids having to fly through the colon in both directions and prevents the occlusion of potential polyps behind haustral folds.
\end{abstract}

\section{Introduction}

Virtual endoscopy is a non-invasive diagnostic procedure aimed at exploring the inner surface of anatomical structures inside the human body. Using advanced image-processing techniques, 3D models are reconstructed from a series of high-resolution 2D images (e.g. CT or MR). A physician can then automatically or interactively navigate through the $3 \mathrm{D}$ virtual model to perform a diagnosis.

One of the most promising uses for virtual endoscopy is the screening of patients for colorectal cancer. This technique mimics conventional colonoscopy with the added advantage that it is less invasive and potentially more desirable as a screening method. An added benefit of virtual colonoscopy is the ability to fly-through the colon in both an antegrade and retrograde direction, significantly increasing the amount of surface displayed. Nevertheless, important sections of the colon often remain obstructed behind haustral folds (see Fig. 1).

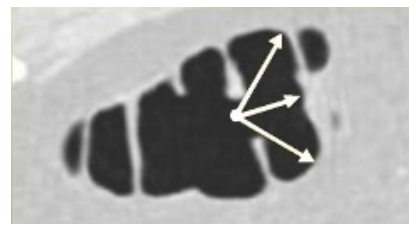

Fig. 1. Limited visibility of a standard virtual camera projection

In this paper we introduce a real-time, interactive visualization technique meant to increase the surface visibility while introducing very little deformation. 


\section{Previous Work}

A number of projections have been proposed in the literature to overcome some of the limitations of the standard perspective volume rendering. These projections seek to display more of the surface of the colon and thereby increase the polyp detection sensitivity.

Serlie et al. 2] propose the use of a cubic viewing space with the virtual camera located in the center of the cube and projecting on each face an image with a 90-degree viewing angle. The cube is then unfolded into a single plane presenting a 360-degree field of view of the colon surface. Off-line animated image sequences are generated in 2] from a number of point samples selected along the central path through the colon. Figure 2 shows a single frame of an animated sequence using this cubing mapping. One of the main drawbacks of this approach is that lesions can be split across several sides of the cube. In addition, the layout is not very natural. It requires to focus on multiple regions of the image to obtain an overall perception of the observed tissues. Similarly, Tiede et al. 6] use the images corresponding to the six faces of the cube to generate precomputed movies known as spherical panoramas.
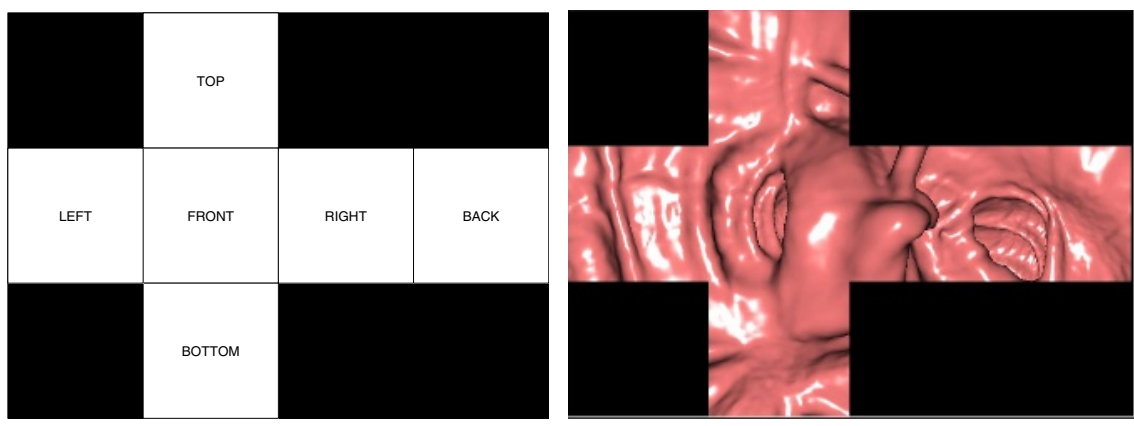

Fig. 2. Six sided unfolded cube projection

Paik et al. 1] suggest the use of map projections that visualize the entire surface of a viewing sphere. Using Mercator and stereographic projections, the surface of the sphere is transformed onto a flat surface. Unfortunately, the distortion introduced by these projections is too high specially near the poles.

Vilanova et. al. [4,3] propose a method to visualize the complete inner colon surface by virtually bisecting the colon along its centerline and unfolding it. Although physicians are unfamiliar with the resulting view, it provides them with a complete overview of the colon in a single image. However, unfolding introduces geometric distortions that can make lesions difficult to identify.

\section{Our Approach}

We propose two panoramic endoscopic projections as variations of the unfolded cubic view. They are designed to obtain a continuous projection, a large field of 

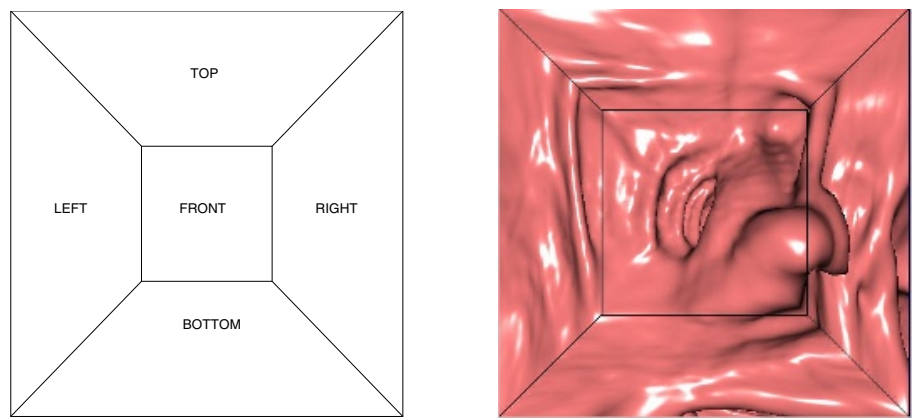

Fig. 3. Panoramic projection of the unfolded cube onto a square
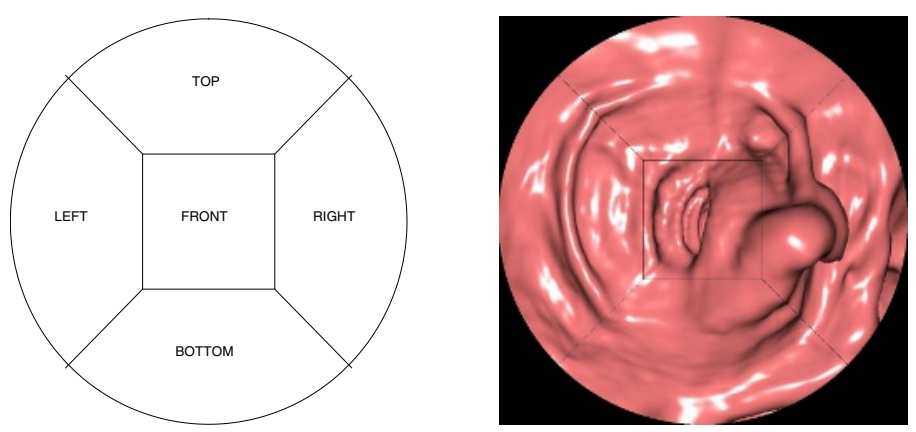

Fig. 4. Panoramic projection of the unfolded cube onto a disk

view, and limited distortion. As in [2, we place the virtual camera in the center of a cubic space and project the images into a single plane using a 90-degree viewing angle. However, instead of using a straightforward unfolding, the front image is mapped into a rectangular window at the center of the plane, the back projection is removed, while the other four images are projected into trapezoidal shaped windows arranged as shown in Figure 3. This approach removes the discontinuities found in [2] and at the same time makes better use of the screen space.

A fast raycast algorithm is used to render each frame in real-time, providing a fully interactive navigation. Figure 3 displays a single frame during a fly-through sequence. The only distortion introduced arises from mapping the cubic walls into trapezoidal views. In order to minimize these distortions, we propose a second projection in which the front face is a mapped into a square while the other four faces are mapped around it into a disk (see Fig. 4).

These projections are designed to guarantee a smooth transition between the five faces. A careful comparison between these projections and the original mapping shown in Figure 2 reveals some distortion closer to the disk periphery. The deformation lessens towards the center of the disk, displaying the frontal view without any deformation whatsoever. 
The size of the frontal view can be adjusted as desired. In the limiting case, when the frontal view covers the entire window, both of these panoramic projections are equivalent to the standard projection with a single virtual camera looking straight ahead.

\section{Projections}

Each endoscopic view is obtained by casting rays from the center of a virtual endoscope positioned inside the volume. The intensity of a point in the endoscopic view is an arbitrary function of the volume intensity values along the corresponding ray. From a given endoscope position, each ray is uniquely characterized by a direction vector. Hence, we model a panoramic view as a vector field (a collection of direction vectors).

In this section, we describe the direction fields corresponding to the proposed panoramic views. These vectors are computed for a fixed endoscope position (we assume the endoscope is placed at the origin of the volume coordinate system and points in the $z$ direction). Arbitrary location and orientation of the endoscope can be taken into account by translation and rotation of the vector field.

The projection is a two-step process. First we find for each point in the panoramic view the corresponding point in one of the faces of the unfolded cube. Then, we find the corresponding direction vector. We focus here on the first step, while the second step is briefly described in Section 4.3

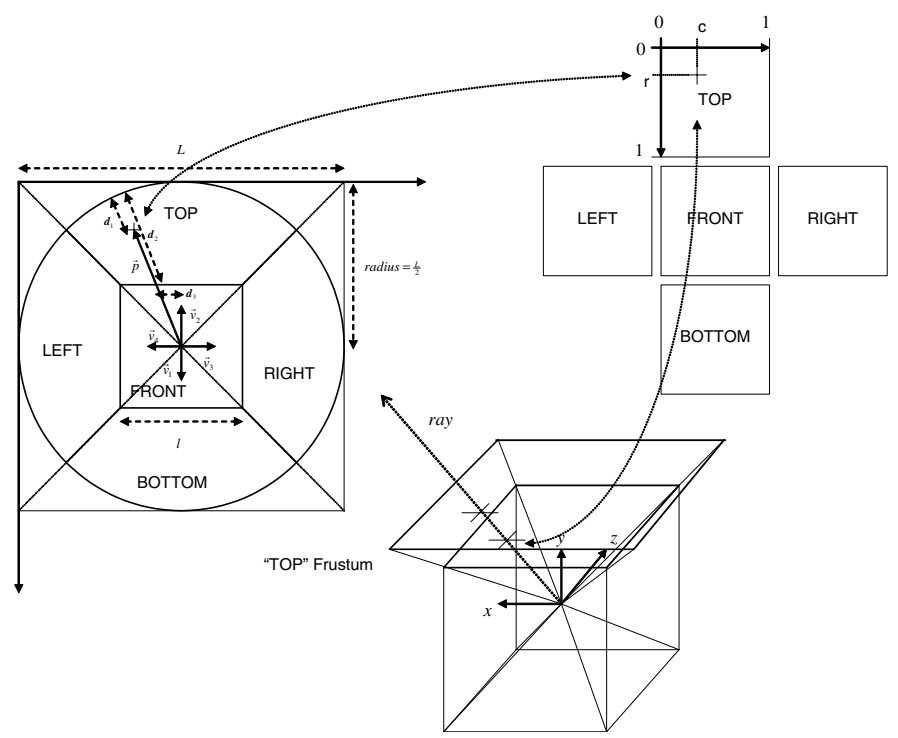

Fig. 5. Determining the coordinates $(r, c)$ on the unfolded cube corresponding to a vector $\vec{p}$ on the disk 


\subsection{Continuous Panoramic Projection of the Unfolded Cube Onto a Disc}

The endoscopic view is modeled as a disk inscribed in a square of side length $L$ (i.e. the disk has a radius equal to $L / 2$ ). Any point inside this square is characterized by a position vector $\vec{p}$ with respect to its center (see Fig. 5 ). The inner square of side length $l$ corresponds to the front view of the unfolded cube. The remaining sections of the disk contain transformed versions of the top, left, right, and bottom views of the unfolded cube.

We introduce four unit vectors $\vec{v}_{1}, \vec{v}_{2}, \vec{v}_{3}, \vec{v}_{4}$. We denote $\vec{v}(i)$ the $i$-th component of a vector $\vec{v}$ and $\|\vec{v}\|$ its norm. The expression $|x|$ denotes the absolute value of a scalar $x$. The operator - denotes the scalar product of two vectors. The scalar product of the vector $\vec{p}$ with $\vec{v}_{i}$ determines the face (bottom, top, right, left, $i=1,2,3,4$ respectively) used during the mapping.

We attach to each face of the unfolded cube a coordinate system (where $(0,0)$ is the upper-left corner of a face and the side length is 1$)$. On a given face, points are located by a set of coordinates $(r, c)$ (see Fig. 5). The mapping between the disk and the cube's faces is given by the following algorithm:

If $\|\vec{p}\|>$ radius Then set the intensity value to zero

Else (the point is inside the disk)

If $\max (|\vec{p}(1)|,|\vec{p}(2)|)<\frac{l}{2}$ Then (the point is in the front view)

$$
\begin{aligned}
r & =0.5+\frac{\vec{p}(1)}{l} \\
c & =0.5+\frac{\vec{p}(2)}{l}
\end{aligned}
$$

Else

$$
\begin{aligned}
& \vec{n}=\frac{\vec{p}}{\|\vec{p}\|} \\
& \text { If } \vec{n} \cdot \vec{v}_{i}>\frac{\sqrt{2}}{2} \text { Then } \\
& \text { cos_angle }=\vec{n} \cdot \vec{v}_{i} \\
& \text { norm_p_inside_front }=\frac{1}{2 \cdot \text { cos_angle }_{-}} \\
& r=\frac{\text { radius }-\|\vec{p}\|}{\text { radius }- \text { norm }_{-} p_{-} \text {inside } \text { front }_{-}}=\frac{d_{1}}{d_{2}} \quad \text { (see Fig. 5) } \\
& \text { End } \\
& c=0.5+\frac{\vec{n}(2)}{2 \cdot \text { cos_angle }_{\text {and }}} \quad\left(\text { note: } \frac{d_{3}}{l}=\left|\frac{\vec{n}(2)}{2 \cdot \cos _{\_} \text {angle }}\right|\right)
\end{aligned}
$$

End

End

\subsection{Continuous Panoramic Projection of the Unfolded Cube Onto a Square}

This view is a variant of the previous approach, where the unfolded cube is mapped onto a square. There is no region left unused, but image distortions are more significant along the diagonals of the endoscopic view (see Fig. 6). The main difference from the computational point of view is the calculation of the $r$ coordinate given below: 


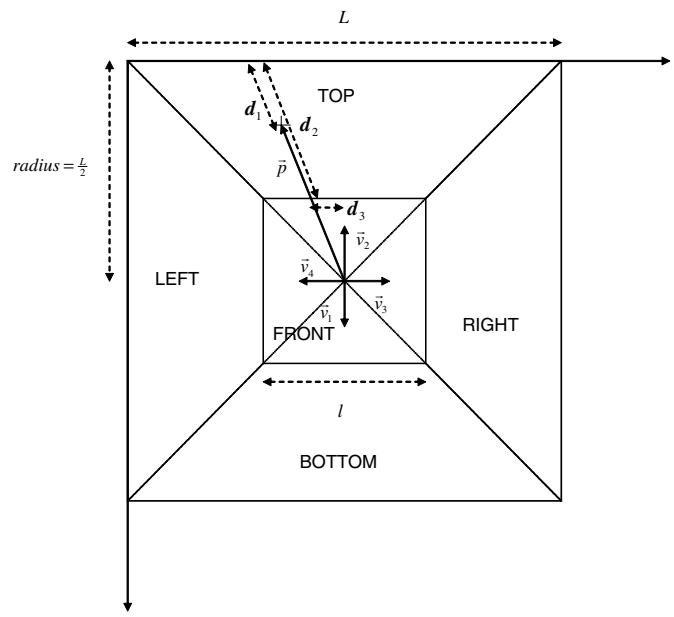

Fig. 6. Determining the coordinates $(r, c)$ on the unfolded cube corresponding to a vector $\vec{p}$ on the square

$$
r=\frac{(\text { radius } / \text { cos_angle })-\|\vec{p}\|}{(\text { radius } / \text { cos_angle })-n_{-} m_{-} p_{-} i n s i d e_{-} \text {front }}=\frac{d_{1}}{d_{2}} .
$$

\subsection{Finding Direction Vectors from Unfolded Cube Coordinates}

To each face of the unfolded cube corresponds a frustum (see Fig. 5 and Fig. 17). A frustum is characterized by a front plane (a square of edge length $l_{\text {front }}$ placed at distance $d_{\text {front }}$ of the endoscope location) and a back plane (a square of edge length $l_{\text {back }}$ placed at distance $d_{\text {back }}$ of the endoscope location).

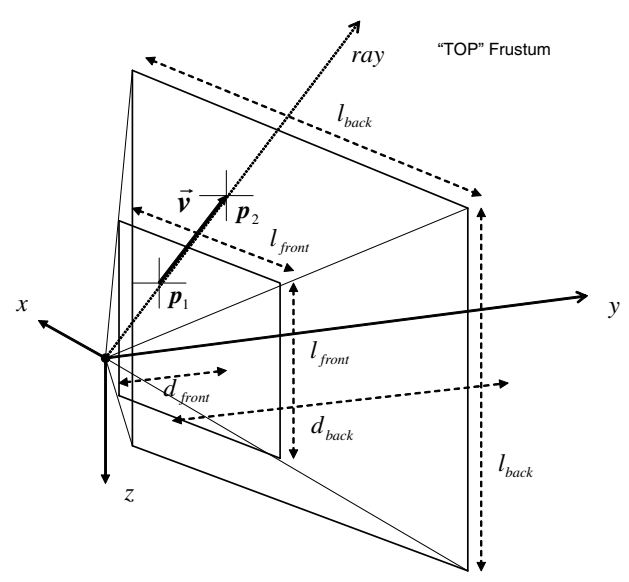

Fig. 7. Finding the direction vector $\vec{v}$ of the ray corresponding to the location $(r, c)$ on the cube faces 
The direction vector $\vec{v}=p_{2}-p_{1}$ of the ray corresponding to the location $(r, c)$ on one of the cube faces is given by:

$$
p_{1}=\left[\begin{array}{c}
\frac{l_{\text {front }}}{2}-c \cdot l_{\text {front }} \\
d_{\text {front }} \\
-\frac{l_{\text {front }}}{2}+r \cdot l_{\text {front }}
\end{array}\right] \text { and } p_{2}=\left[\begin{array}{c}
\frac{l_{\text {back }}}{2}-c \cdot l_{\text {back }} \\
d_{\text {back }} \\
-\frac{l_{\text {back }}}{2}+r \cdot l_{\text {back }}
\end{array}\right] .
$$

Most of the previous operations can be combined to obtain a more compact and efficient implementation. The symmetry of the vector field can also be exploited to reduce the computational cost.

\section{Results}

Figure 8 shows a side by side comparison of the amount of the colon surface visualized using a standard camera projection and the proposed panoramic projections. Voxels shown in red correspond to the areas not visible after a one-way flight through the virtual colon. Since the panoramic projections show simultaneously a forward, left, right, up and down views, we get nearly $100 \%$ of surface coverage with one single pass [5]. To achieve better coverage, a conventional virtual navigation typically executes an additional fly-through in the reverse direction. The added flight not only increases the reading time as some parts of the surface are examined twice, but even then some areas remain hidden behind haustral folds. The panoramic projections therefore could be used to speed up the $3 \mathrm{D}$ reading as we can cut the fly-time in half.
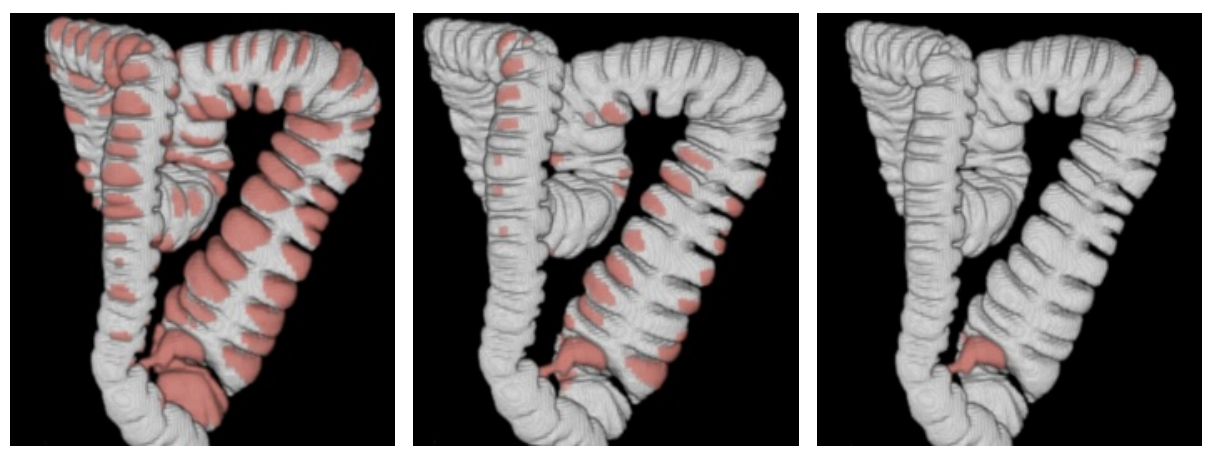

Fig. 8. Global view of the colon after a one-way flight using a standard perspective projection (left), a two-way flight using a standard projection (middle), and a one-way flight using a panoramic projection (right)

Our implementation allows a real-time fly through and, as discussed before, the geometry of the projection limits distortion. User studies are underway to compare the detection rate, speed and general acceptance of the proposed projections. Preliminary results seem very positive, as features tend to stay in view 
for longer periods of time. The proposed projections are not restrictive to the colon and therefore can potentially be used to display the inner surface of any virtual organ.

\section{References}

1. Paik, D., Beaulieu, C., Jeffrey, R., Karadi, C., Napel, S.: Visualization Modes for CT Colonography Using Cylindrical and Planar Map Projections. J. Comput. Assist. Tomography, 24:179-188, 2000.

2. Serlie, I., Vos, F., van Gelder, R., Stoker, J., Truyen, R., Gerritsen, F., Nio. Y., Post, F.: Improved Visualization in Virtual Colonoscopy Using Image-Based Rendering, Proccedings of IEEE TCVG Symposium on Visualization, 137-146, 2001.

3. Vilanova Bartrolí, A., Gröller, E.: Geometric Modelling for Virtual Colon Unfolding, in Geometric Modeling for Scientific Visualization, 453-468, Springer-Verlag, 2003.

4. Vilanova Bartrolí, A., Wegenkittl, R., König, A., Gröller, E.: Nonlinear Virtual Colon Unfolding. IEEE Visualization Conference Proceedings, 411-418, 2001.

5. Wax, M., Kreeger, K., Anderson, J.: Endoscopic View in Virtual Colonoscopy: Achieving Complete Surface Visualization, RSNA, 2001.

6. Tiede, U., Sternberg-Gospos, N., Steiner, P., Höhne, K. H., Virtual Endoscopy using Spherical QuickTime-VR Panorama Views, in J. Westwood et al. (eds.): Medicine meets Virtual Reality, Studies in Health Technology and Informatics 85, IOS Press, Amsterdam, 2002, 523-528. 